International Journal of Applied Dental Sciences 2021; 7(2): 558-560

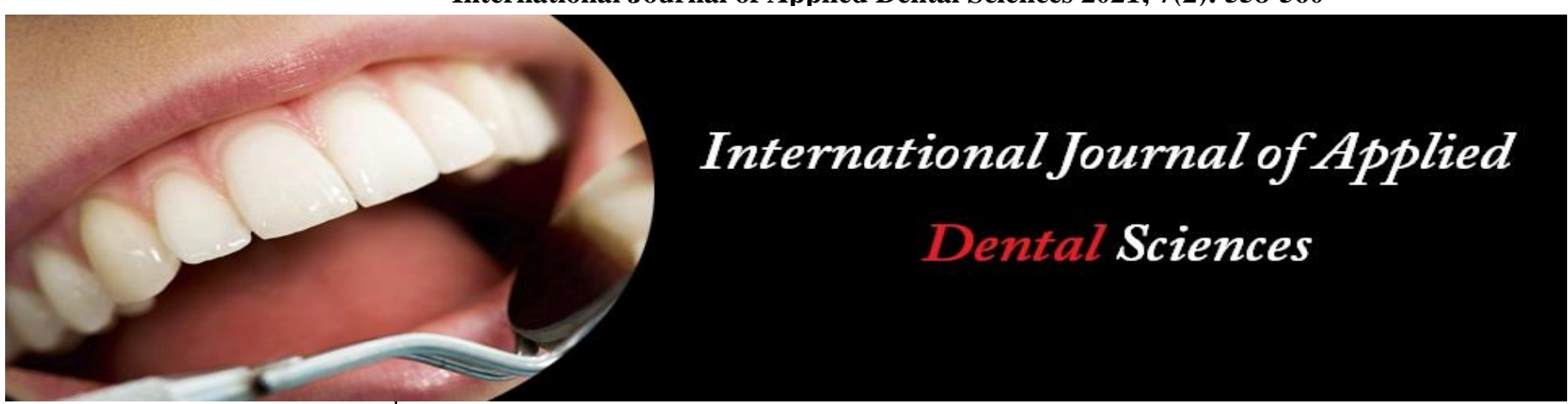

ISSN Print: 2394-7489

ISSN Online: 2394-7497

IJADS 2021; 7(2): 558-560

(C) 2021 IJADS

www.oraljournal.com

Received: 04-02-2021

Accepted: 06-03-2021

\section{Lalit Kharad}

Salman Dental Center, Building 90c Road 902. Block 109, HiddAl Muharraq, Kingdom of

Bahrain, Bahrain
Corresponding Author: Lalit Kharadi

Salman Dental Center, Building 90c Road 902. Block 109, HiddAl Muharraq, Kingdom of Bahrain, Bahrain

\section{Applications of mini-implants in orthodontics}

\section{Lalit Kharadi}

\section{DOI: https://doi.org/10.22271/oral.2021.v7.i2i.1262}

\section{Abstract}

(Salman Dental Center, Hidd) Mini-implants obtain anchorage form bone and thus provide increased control of orthodontic tooth movement and help in reducing the side effects during the management of complex malocclusion. Since the introduction of mini-implants in orthodontics, is has undergone several modifications in design, composition, size and shape. In this article, a wide-ranging review of the current application of mini-implants, the success rates of mini-implants, factors affecting the success rates of mini-implants, complications with mini-implants has been described. The utility of mini-implants has increased in the recent years. With the high success rates found with palatal mini-implants, mini-screw assisted rapid palatal expansion is popular method in contemporary orthodontics. Additionally, miniimplants with aligners can also be used to provide an esthetic option to the patients for the correction of complex malocclusion.

Keywords: mini-implants, orthodontics; temporary anchorage devices (TADs)

\section{Introduction}

Anchorage in orthodontics has been a problem since the inception of modern orthodontics by Dr. Edward Angle. Anchorage refers to the prevention of unwanted tooth movement. One of the concerns regarding anchorage is that it is difficult to obtain adequate anchorage from the posterior teeth while moving the anterior teeth to the maximum capacity. In cases where maximum retraction of anterior teeth is required, absolute anchorage with mini-implants can be beneficial. When mini-implants were introduced to the orthodontic world, the protocol regarding implants were not established and this led to a higher level of failure. However, in the recent times, the success rates have been found to be pretty high. This is due to the developments in the design, size, and shape of mini-implants. There is a good amount of heterogeneity in the mini-implant design with diameter from $1 \mathrm{~mm}$ to $2.4 \mathrm{~mm}$ and length from $4 \mathrm{~mm}$ to $21.5 \mathrm{~mm}^{[1,2]}$

The applications of mini-implants are not only limited to retraction of anterior teeth in cases requiring maximum anchorage. Mini-implants are also useful for protraction of molars, expansion of maxillary arch, management of impacted canines, intrusion of posterior maxillary and mandibular dentition, and correction of inclined occlusal planes ${ }^{[1-5]}$.

\section{Materials and Methods}

\subsection{Application of Mini-implants in orthodontics}

Mini-implants are used widely in orthodontics for various purposes. One of the major advantages of mini-implants is that it allows for maintenance of good oral hygiene, and has a reduced incidence of periodontal problems as compared to conventional anchorage devices such as Nance appliance ${ }^{[6]}$. The utility of mini-implants to orthodontists because of easily insertion and the widespread acceptance of the mini-implants by the patients has led to increased popularity of mini-implants. There have been considerable developments in the field of orthodontic mini-implants mainly in the advances in design, advances in placement techniques, and a better understanding of the risk factors. In comparison with previous anchorage systems such as mini-plates, mini-implants are less bulky and thus can be inserted in areas between the roots of teeth, or in alveolar bone. Mini-implants have found to be successful in the range of 80 to 100 percentage. The buccal mini-implants have shown lower success rates than palatal mini-implants.

$$
\sim 558 \sim
$$


Thus, the use of palatal mini-implants have increased in past few years ${ }^{[3]}$. The failure rates of mini-implants are in the low range of 10 to 30 percentage $[1,7,8]$. The factors that are associated with the failure of mini-implants are the diameter of the screw, the age of the patient, thickness of cortical bone, density of bone, type and depth of soft-tissue, inflammation, oral hygiene, preparation of the insertion area and primary stability ${ }^{[8]}$. The thickness of cortical bone is important for the success of mini-implants, but the thickens of cancellous bone is not critical ${ }^{[9,10]}$. Another critical factor for the success of mini-implants is the type of forces applied on mini-implants and the adhesives used for the appliance cementation. If heavy forces are used in rotational movement, then it may increase the chances of failure of mini-implants. On the other hand, the advances in the adhesives in dentistry has led to increase in the bond strength and decrease in the failure rate of the orthodontic appliances on the teeth ${ }^{[11-13]}$. Such advances are critical for the success of mini-implants.

\subsection{Mini-implants and anterior open-bite}

Mini-implants can be used in anterior open bite patients for the correction of vertical discrepancies. In such cases, miniimplants can be used in the posterior arch between the maxillary molars and intrusive force can be applied on the posterior dentition through the mini-implants ${ }^{[6]}$. This leads to intrusion of posterior teeth and thus, closure of mandible by autorotation thus closing the anterior open bite ${ }^{[14]}$. Miniimplants can also be inserted in the mandibular arch between the mandibular molars. Mandibular mini-implants are helpful in establishing an intrusive force on the mandibular molars to prevent mandibular molar extrusion while the maxillary molars are intruded ${ }^{[15]}$.

\subsection{Mini-implants and maxillary expansion}

Mini-implants are often placed in the palatal bone for expansion of maxillary arch to correct the posterior crossbite. ${ }^{[16,17]}$ Rapid palatal expansion appliances using miniimplants for anchorage are known as mini-screw assisted rapid palatal expansion (MARPE) or bone-anchored maxillary expansion appliances. MARPE appliance are used so that more skeletal expansion is achieved and dental expansion is minimal. Expansion with MARPE increased the maxillary arch width and thus, leads to resolution of posterior crossbite. It has also shown to cause increase in airway volume after expansion ${ }^{[16,17]}$. The changes achieved with MARPE are found to be stable over time. In a recent study, Mehta et al. showed that MARPE appliances led to an increased nasopharyngeal volume after 2.5 years following expansion [3]. However, there were no differences in the total airway volume between MARPE and controls. With conventional maxillary expansion, bilateral expansion of maxillary arch occurs even in unilateral crossbite patients. With the help of mini-implants, new designs such as Unilateral MARPE UMARPE have been developed for the correction of unilateral crossbite ${ }^{[18]}$.

\subsection{Mini-implants and distalization of molars}

In Class III malocclusion, mandibular dentition is placed anteriorly in relation to the maxillary arch ${ }^{[19]}$. In such cases, mini-implants can be place in the buccal shelf area used to distalization of mandibular arch. Such mini-implants are known as buccal shelf mini-implants. In addition, miniimplants can also be placed on the mandibular ramus for the uprighting of mandibular second molars. Chang et al. showed that ramus shelf-mini-implants can be used efficiently for achieving uprighting of mandibular molars ${ }^{[20]}$.

\subsection{Mini-implants and Aligner therapy}

Clear aligner therapy has an important role in contemporary orthodontics [21, 22]. As more and more adult patients are undertaking orthodontic treatment, the emphasis for esthetic treatment modalities has increased. In addition to being esthetically pleasing, clear aligners have the advantage of applying low forces on the teeth, and thus can potentially lead to decreased root resorption as compared to fixed orthodontic appliances ${ }^{[23]}$. Over the years, the type of malocclusions that can be treated with clear aligners has increased because of the use of mini-implants with aligners. Mini-implants can be inserted between the maxillary anterior teeth to intrude the maxillary anteriors in conjunction with aligner therapy. ${ }^{[24]}$ In addition, many patients with Class III malocclusion can be treated with mini-implants and aligner therapy to distalize the mandibular arch with mini-implants.

\section{Results \& Discussion}

The insertion angle of mini-implants has an impact on the type of force and the direction of force applied on miniimplants. This has an effect on the success of mini-implants. When mini-implants are inserted between the roots of teeth, care has to be taken not to cause injury to the adjacent teeth. This can be evaluated with radiographs before inserting the mini-implants. It has been shown that 2-dimensinoal (2D) radiographs are not as effective in evaluating anatomy or head and neck surfaces as 3-dimensional (3D) radiographs such as Cone-beam computed tomography (CBCT) ${ }^{[25]}$. Furthermore, it has been shown that rotations errors in the position of head while recording the radiographs can lead to errors in measurements made on the $2 \mathrm{D}$ radiographs ${ }^{[26]}$. It has been shown that mini-implants that are angle while inserting have a higher contact area with the bone and thus, have increased success rates ${ }^{[27]}$. However, it should be noted that very high angle may create slippage while inserting the mini-implant. ${ }^{[27]}$

\subsection{Complications of mini-implants}

A very common complication of mini-implants is mobility. Mobility can be considered failure if it is too high to warrant removal of mini-implant. Sometimes mini-implants also undergo fracture. In such cases, the mini-implant should be retrieved safely with orthodontic instruments taking care that the patient does not swallow the mini-implants ${ }^{[7,27]}$. When there is a failure of mini-implants, a second mini-implant can be inserted in the same area after 3 months or in a difference area. Studies have reported that mini-implants with reduced diameter or length have a higher chance of failure. In addition, patients who are chronic smokers have a higher chance of peri-implantitis leading to mobility of miniimplants and failure of mini-implants ${ }^{[28]}$.

\section{Conclusions}

Mini-implants have a wide range of applications for orthodontic treatment. The most important advantage of miniimplants is that it provides absolute anchorage. The improvements in the design of mini-implants and advancements in the understanding of the risk factors have led to high success rates of mini-implants. Mini-implants can be used successfully for intrusion, expansion, and distalization, with fixed orthodontic appliances and in conjunction with clear aligner therapy. 


\section{References}

1. Crismani AG, Bertl $\mathrm{MH}$, Celar AG, Bantleon HP, Burstone CJ. Miniscrews in orthodontic treatment: review and analysis of published clinical trials. Am J Orthod Dentofacial Orthop 2010;137(1):108-113. doi:10.1016/j.ajodo.2008.01.027.

2. Manni A, Cozzani M, Tamborrino F, De Rinaldis S, Menini A. Factors influencing the stability of miniscrews. A retrospective study on 300 miniscrews. Eur J Orthod. 2011;33(4):388-95. doi: 10.1093/ejo/cjq090. Epub 2010 Oct 6. PMID: 20926556.

3. Mehta S, Wang D, Kuo CL, Mu J, Vich ML, Allareddy V et al. Long-term effects of mini-screw-assisted rapid palatal expansion on airway. Angle Orthod. 2020;10.2319/062520-586.1. doi:10.2319/062520-586.1.

4. Türköz C, Ataç MS, Tuncer C, Balos Tuncer B, Kaan E. The effect of drill-free and drilling methods on the stability of mini-implants under early orthodontic loading in adolescent patients. Eur J Orthod 2011;33(5):533-536. doi:10.1093/ejo/cjq115.

5. Chang J, Mehta S, Chen PJ, Upadhyay M, Yadav S. Correction of open bite with temporary anchorage device-supported intrusion. APOS Trends in Orthodontics 2019;9(4):246-251.

doi: 10.25259/APOS_101_2019.

6. Kim SH, Hwang YS, Ferreira A, Chung KR. Analysis of temporary skeletal anchorage devices used for en-masse retraction: a preliminary study. Am J Orthod Dentofacial Orthop 2009;136(2):268-276.

doi:10.1016/j.ajodo.2007.08.023.

7. Miura K, Motoyoshi M, Inaba M, Iwai H, Karasawa Y, Shimizu N. A preliminary study of the effects of lowintensity pulsed ultrasound exposure on the stability of orthodontic miniscrews in growing rats. Eur J Orthod. 2014;36(4):419-424. doi:10.1093/ejo/cjt066.

8. Chang JZ, Chen YJ, Tung YY et al. Effects of thread depth, taper shape, and taper length on the mechanical properties of mini-implants. Am J Orthod Dentofacial Orthop.

2012;141(3):279-288. doi:10.1016/j.ajodo.2011.09.008.

9. Liu TC, Chang CH, Wong TY, Liu JK. Finite element analysis of miniscrew implants used for orthodontic anchorage. Am J Orthod Dentofacial Orthop 2012;141(4):468-476. doi:10.1016/j.ajodo.2011.11.012.

10. Laursen MG, Melsen B, Cattaneo PM. An evaluation of insertion sites for mini-implants: a micro - CT study of human autopsy material. Angle Orthod 2013;83(2):222229. doi:10.2319/042512-344.1.

11. Mehta S, Mehta F, Patel R, Kumar A. Effect of Repeated Bonding on the Shear Bond Strength of Smartbond Cyanoacrylate Orthodontic Adhesive. Indian Journal of Orthodontics and Dentofacial Research 2016;2(1):14-18.

12. Gange P. The evolution of bonding in orthodontics. Am J Orthod Dentofacial Orthop. 2015;147(4):S56-S63. doi:10.1016/j.ajodo.2015.01.011.

13. Mehta F, Mehta S, Kumar A, Gandhi V. Effect of Repeated Bonding on the Shear Bond Strength of Transbond XT (3M Unitek). IOSR J Dent Med Sci 2016;15(5):14-21.DOI: 10.9790/0853-1505071421.

14. Deng JR, Li YA, Wang XD, Li J, Ding Y, Zhou YH, et al. Evaluation of long-term stability of vertical control in hyperdivergent patients treated with temporary anchorage devices. Curr Med Sci 2018;38(5):914-919. doi:10.1007/s11596-018-1962-6.

15. Scheffler NR, Proffit WR, Phillips C. Outcomes and stability in patients with anterior open bite and long anterior face height treated with temporary anchorage devices and a maxillary intrusion splint. Am J Orthod Dentofacial Orthop 2014;146(5):594-602. doi:10.1016/j.ajodo.2014.07.020.

16. Abu Arqub S, Mehta S, Iverson MG, Yadav S, Upadhyay M, Almuzian M. Does Mini Screw Assisted Rapid Palatal Expansion (MARPE) have an influence on airway and breathing in middle-aged children and adolescents? A systematic review. Int Orthod. 2021;19(1):37-50. doi: 10.1016/j.ortho.2021.01.004. Epub 2021 Jan 28. PMID: 33516650 .

17. Kabalan O, Gordon J, Heo G, Lagravère MO. Nasal airway changes in bone-borne and tooth-borne rapid maxillary expansion treatments. Int Orthod 2015;13(1):115. doi:10.1016/j.ortho.2014.12.011.

18. Dzingle J, Mehta S, Chen PJ, Yadav S. Correction of Unilateral Posterior Crossbite with U-MARPE. Turk J Orthod 2020;33(3):192-196. Published 2020 Jul 20. doi:10.5152/TurkJOrthod.2020.20034.

19. Kuroda S, Sugawara Y, Deguchi T, Kyung HM, TakanoYamamoto T. Clinical use of miniscrew implants as orthodontic anchorage: success rates and postoperative discomfort. Am J Orthod Dentofacial Orthop 2007;131(1): 9-15. doi:10.1016/j.ajodo.2005.02.032.

20. Lin SY, Chang CH, Roberts WE. Simple mechanics to upright horizontally impacted molars with ramus screws. Int J Ortho Implantol 2015;40:84-92.

21. Mehta F, Mehta S. Aligners: The Rapidly Growing Trend in Orthodontics Around the World. Indian J Basic Appl Med Res 2014;3(4):402-409.

22. Chan E, Darendeliler M. The Invisalign appliance today: A thinking persons orthodontic appliance. Semin Orthod. 2017;23:12-64.

23. Gandhi V, Mehta S, Gauthier M, Mu J, Kuo CL, Nanda $\mathrm{R}$ et al. Comparison of external apical root resorption with clear aligners and pre-adjusted edgewise appliances in non-extraction cases: a systematic review and metaanalysis. Eur J Orthod 2021;43(1):15-24. doi:10.1093/ejo/cjaa013.

24. Ravera S, Castroflorio T, Garino F, Daher S, Cugliari G, Deregibus A. Maxillary molar distalization with aligners in adult patients: a multicenter retrospective study. Prog Orthod 2016;17:12. doi:10.1186/s40510-016-0126-0.

25. White SC, Pharoah MJ. Oral radiology principles and interpretation. 5th ed. St Louis: Mosby 2004.

26. Mehta S, Dresner R, Gandhi V, Chen PJ, Allareddy V, Kuo CL et al. Effect of positional errors on the accuracy of cervical vertebrae maturation assessment using CBCT and lateral cephalograms. J World Fed Orthod 2020;9(4):146-154. doi:10.1016/j.ejwf.2020.09.006.

27. Wilmes B, Panayotidis A, Drescher D. Fracture resistance of orthodontic mini-implants: a biomechanical in vitro study. Eur J Orthod 2011;33(4):396-401. doi:10.1093/ejo/cjq151.

28. Dalessandri D, Salgarello S, Dalessandri M et al. Determinants for success rates of temporary anchorage devices in orthodontics: a meta-analysis $(n>50)$. Eur $\mathbf{J}$ Orthod 2014;36(3):303-313. doi:10.1093/ejo/cjt049. 